\title{
Digital Variance Angiography: A Promising Alternative Technology to Traditional Angiography for Improvement of Image Quality with Reduction of Radiation and Contrast Medium Doses
}

\author{
Zhonghua $\operatorname{Sun}^{1}$ iD
}

Received: 26 October 2020/ Accepted: 2 December 2020/Published online: 3 January 2021

(C) Springer Science+Business Media, LLC part of Springer Nature 2021

Digital subtraction angiography (DSA) is the standard method for imaging diagnosis and therapeutic treatment of cardiovascular disease. However, the high radiation dose and large amount of contrast medium associated with DSA present risk of radiation-induced malignancy and contrastinduced nephropathy. It is necessary to develop image post-processing approaches to improve image quality of DSA while reducing radiation and contrast medium doses. Digital variance angiography (DVA), a new image processing method, shows potential to address these issues by improving vessel visualization through use of kinetic imaging principle to obtain more information from the raw angiographic data.

The clinical research team from Heart and Vascular Centre in Hungary recently reported promising results of this technique in their preliminary studies [1-3]. Researchers evaluated the diagnostic value of DVA compared with standard DSA in a small group of patients undergoing lower extremity angiographic examinations. Based on single- and multi-center reports, they found that DVA offered image quality by 2.3 to 3.3 times of signal-tonoise ratio (SNR) than that of DSA images. Furthermore, qualitative comparison of image quality between DVA and DSA images indicated that DVA improved image quality in up to $73 \%$ of all vascular regions with significant interobserver agreement [1-3]. For lower limb interventional procedures among 4 patients with peripheral arterial disease, DVA images were evaluated as the preferred ones in

Zhonghua Sun

z.sun@curtin.edu.au

1 Discipline of Medical Radiation Sciences, Curtin University, Perth, WA 6845, Australia more than $80 \%$ of all comparisons in terms of both diagnostic and therapeutic values [3].

In this issue of CVIR, Bastian et al. further tested this promising technique in 12 patients with metal implants and reported its clinical value by creating images with significantly improved image quality [4]. A total of 85 raw lower limb angiographic image pairs (40 contained metal implants and 45 pairs without implants) were postprocessed using Kinetic Medical Imaging Tool and Syngo software. Contrast-to-noise ratio (CNR) was measured and compared between DVA and DSA images, while the ratio of $\mathrm{CNR}_{\mathrm{DVA}} / \mathrm{CNR}_{\mathrm{DSA}}$ was calculated to determine whether DVA outperforms DSA. Their results showed the median $\mathrm{CNR}_{\mathrm{DVA}} / \mathrm{CNR}_{\mathrm{DSA}}$ was 1.84 , with overall preference of DVA images ranked in $84.5 \%$ of all images with significant inter-observer agreement $(p<0.001)$. Three radiologists randomly assessed 170 DVA and DSA images using a 5-point Likert scale. Significantly higher scores were found in DVA than DSA images (4.43 \pm 0.06 vs $3.64 \pm 0.08$, $p<0.001)$ in all groups, regardless of 'Implant' or 'no Implant' images [4].

There are three observations from Bastian's study that bear discussion. First, the authors have further confirmed the clinical value of DVA technology by improving image quality even in the presence of metal implants in these patients. This could contribute to reducing radiation dose in future studies. Second, despite being the first report of applying DVA in this area, the small sample size is one of the limitations. Furthermore, authors did not provide details on the volume of iodinated contrast medium used in the angiographic examinations. Their previous reports showed that DVA significantly improved image quality by up to 4.5 times SNR than DSA in lower extremity carbon dioxide $\left(\mathrm{CO}_{2}\right)$ angiography $[2,3]$, highlighting the 
potential use of $\mathrm{CO}_{2}$ as a safe contrast medium. A recent study validated the feasibility and safety of using $\mathrm{CO}_{2}$ as a contrast agent to protect renal function in the midterm outcomes of endovascular repair of aortic aneurysm through comparing $\mathrm{CO}_{2}$-guided angiography with iodinated contrast-angiography patients [5]. The third observation is related to the clinical applicability of these results to other clinical sites with inclusion of different cardiovascular areas. Currently, these findings primarily come from the same clinical group with use of specific software tool for generation of DVA images. Further research should aim to test this innovative technology in more clinical centers with applications extended to areas beyond the lower limb angiographic region.

In summary, Bastian and colleagues have successfully demonstrated the superiority of DVA over standard DSA in the assessment of image quality of lower limb angiography with metal implants. However, since the current study along with other reports from the same group is based on a small number of patients and is limited to lower extremity angiographic examinations, large-scale studies, preferably multicenter studies, are needed to validate its clinical value before recommending the use of DVA in routine angiographic practice.

Funding No funding is available.

\section{Compliance with Ethical Standards}

Conflict of interest The author has declared no conflict of interest.

\section{References}

1. Gyano M, Gog I, Orias VI, Ruzsa Z, Nemes B, Csobay-Novak C, Olah Z, Nagy Z, Merkely B, Szigeti K, Osvath S, Sotonyi P. Kinetic imaging in lower extremity arteriography: Comparison to digital subtraction angiography. Radiology. 2019;290:246-53.

2. Orias VI, Gyano M, Gog I, Szollosi D, Veres DS, Nagy Z, CsobayNovak C, Zoltan O, Kiss JP, Osvath S, Szigeti K, Zoltan R, Sotonyi P. Digital variance angiography as a paradigm shift in carbon dioxide angiography. Invest Radiol. 2019;54:428-36.

3. Gyano M, Csobay-Novak C, Berczeli M, Gog I, Kiss JP, Szigeti K, Osvath S, Nemes B. Initial operating room experience with digital variance angiography in carbon dioxide-assisted lower limb interventions: A pilot study. Cardiovasc Interv Radiol. 2020;43:1226-31.

4. Bastian MB, Konig A, Viniol S, et al. Digital variance angiography in lower limb angiography with metal implants. Cardiovasc Intervent Radiol. 2020. https://doi.org/10.1007/s00270-020-026566.

5. Takeuchi Y, Morikage N, Matsuno Y, Nakamura T, Samura M, Ueda K, Harada T, Ikeda Y, Suehiro K, Ito H, Sakata K, Hamano K. Midterm outcomes of endovascular aortic aneurysm repair with carbon dioxide-guided angiography. Ann Vasc Surg. 2018;51:170-6.

Publisher's Note Springer Nature remains neutral with regard to jurisdictional claims in published maps and institutional affiliations. 